\title{
Reading, Processing and Interacting with Hypertext on the Web
}

\author{
Gemma Fitzsimmons, Mark J Weal, Denis Drieghe \\ University of Southampton \\ Southampton, UK \\ \{G.Fitzsimmons, mjw, D.Drieghe\}@soton.ac.uk
}

\begin{abstract}
We increasingly spend a vast amount of time on the Web and much of that time is spent reading. One of the main differences between reading non-Web based text and reading on the Web is the presence of hyperlinks within the text, linking various related Web content and Web pages together. Some researchers and commentators have claimed that hyperlinks hinder reading because they are a distraction that may have a negative effect on the reader's ability to process the text. However, very few controlled experiments have been conducted to verify these claims. In the experiments documented here we utilise eye tracking as a new methodology for examining how we read hyperlinked text. There is a well-documented tight link between when and where we look and what we process. Eye movements have been used extensively to help us to understand the cognitive processing that occurs during reading, but there has been very little research into how our reading differs when we read information on the Web.
\end{abstract}

Keywords: Hyperlinks; Reading; Skim reading; Web Science; Psychology; HCl; Eye movements

\section{INTRODUCTION}

The Web has opened up access to information and had a profound influence upon the lives of countless people. Users of the Web engage in a wide variety of different activities, such as searching for information, reading the news, sending and receiving email and social networking, to name just a few [1]. Arguably, within the majority of these activities, the primary task that users engage in is reading text in some shape or form. Though there has been extensive research examining reading behaviour [2,3], which will be discussed here, reading online text (termed hypertext [4]) has some important differences to reading plain text that is not in a Web environment (such as a printed novel). One of the main differences between online reading and offline reading is the presence of hyperlinks.

Hyperlinks are prolific online, either embedded in the text or listed in a sidebar as part of a navigation system. Hyperlinks are distinguished from the rest of the onscreen text by being displayed in a different colour (often blue) and are often underlined. Any piece of text or image can be made a hyperlink that once clicked will display the appropriate linked content. The research that has been conducted to date has only scratched the surface of how we read hypertext. The primary goal of the present thesis is therefore to develop a theoretical framework explaining how we read, interact with and process hypertext.

By studying how people read hypertext on the Web, it will then be possible to generate suggestions for how to design and setup Web pages in a way that most suits the demands and limitations of the human information processing system.

\section{RESEARCH QUESTIONS}

\subsection{Does the Saliency of Hyperlinks have a Negative Influence on Reading for Comprehension?}

We begin by only focusing on participants reading static Webpages where they will not be able to click on hyperlinks and navigate the Web. By removing the ability to click and focusing on the reading behaviour we can tease apart the differences between reading on the Web and traditional reading and explore the impact of each of these differences. Our first question focuses on the impact of having salient, coloured words as hyperlinks and whether this saliency has a negative or distracting effect on reading behaviour.

\subsection{How Does Hypertext Affect the Importance of the Content?}


We need to consider the impact having hyperlinks in the text has on the importance of the text. Do hyperlinks effect the way we read and perceive the text, and do we judge hyperlinks as signals to inform us where important sections of text are located?

\subsection{How Does Skim Reading Affect the Reading and Comprehension of Webpages and What is the Influence of Hyperlinks on Skim Reading?}

Next, we need to consider task effects during reading. It is not always possible to read for comprehension, especially when there is a large amount of information to read through. With this in mind we explore the impact of hyperlinks and task effects on reading behaviour and comprehension.

\subsection{How Does Clicking and Navigating Hypertext Affect Reading Behaviour and Comprehension?}

Finally, once we have the basic results, we can then allow participants to click and navigate through Webpages and observe what additional impact being able to navigate has on the effects already observed reading static Webpages.

\section{METHODLOGY}

\subsection{Biology of the Eye}

When we make eye movements, the movements themselves are called saccades. In between these saccades our eyes are relatively still, which is called a fixation. We take in information during fixations. Saccades are necessary due to the anatomy of the eye and more specifically the retina. The retina contains a very high concentration of photoreceptors called rods and cones. Cones are necessary for high visual acuity. The majority of the cones in the retina are in an area called the fovea which covers about $1^{\circ}$ degree of visual angle on either side from the fixation point. Moving beyond the fovea, there is a large reduction in the number of cones and therefore a high acuity drop off. Readers can still obtain some visual information from the parafovea (an area which extends to about $5^{\circ}$ degree of visual angle either side of the fovea), but it does not have as high acuity as the fovea. Beyond the parafovea is peripheral vision which has low acuity and readers may only be aware of relatively low level information, such as word length and spaces during reading.

In order to read we need the words to be focused on by the high acuity fovea to be able to process them. Therefore, we need to move our eyes so that the fovea can be utilised to take in the most visual information while reading. It has also been observed that the more difficult the text is the longer the fixations are and the shorter the saccades and there is also more re-reading occurring [5].

\subsection{Eye Tracking Technology}

The eye tracker used for the experiments within this thesis is the EyeLink 1000, which records the eye position every millisecond and has an accuracy of $0.25^{\circ}-0.5^{\circ}$ of visual angle. By using infrared light directed at the participant and a camera to record the light reflected back, the eye position can be calculated.

\subsection{Eye movements are linked to online cognitive processing.}

Recording eye movements is an objective way of collecting data about behaviour and a number of studies have shown that eye movements provide an unobtrusive, real-time behavioural index of visual and cognitive processing [2-3,6]. It has also been observed that when eye movements are absent in an individual their visual perception can be surprisingly normal. For example, an individual referred to as $\mathrm{Al}$ who was born without the ability to make eye movements and who compensates for this lack of eye movements by making saccadiclike movements of the head. This suggests that saccadic movements of the eye, or head in this case, form an optimal sampling method for the brain. The head movements made by $\mathrm{Al}$ were present not only in reading, but also in visual scene viewing, orientating her head movements to locations of interest in the scenes [7].

This kind of orientating of eye movements to locations of interest in a scene was first observed by showing that different eye movement patterns were observed dependent on the task being completed. Participants viewed a painting by Repin entitled "An Unexpected Visitor" that depicts a family in the room with another person entering the room. The participants were given different instructions while observing the picture and having their eyes tracked. A different pattern of eye movements was observed for the different instructions. For example, when asked to remember the clothes worn by the people in the room, the participants focused on the people in the room and looked at the whole person to observe all of their clothes. However, when asked to estimate the age of the people in the room, the participants tended to focus on the faces of the people in the room [8]. This suggests that the eye movements are driven by the task at hand. Together, this evidence, along with many other studies [for a review see 2,3] points to the notion that eye movements can be used to gain an insight into cognitive processes. 


\section{LITERATURE REVIEW}

The literature for this thesis is mostly based in the fields of psychology and human computer interaction. The main methodology in this thesis is eye tracking using eye movements to understand how individuals interact with text and on the Web.

\subsection{Reading}

The eye movement methodology has been used extensively to investigate how individuals process text during reading. There are a number of factors to consider that influence when and where we move our eyes and investigating these factors can tell us a great deal about how text is processed during reading.

\subsubsection{Where we move our eyes}

Research has shown that the decision of where to move the eyes is largely driven by low-level cues such as word length and space information. If the word to the right of fixation is a long word of 9 characters, the next saccade will be longer than if it were a medium sized 5-character word [for a review, see 2]. This reflects the tendency of the system to land on a position in the word that is optimal for recognising this word, which will be more character positions into a long word compared to a short word. Also spaces between words are used as cues to where the word ends and can be used to help target saccades. When spaces are removed reading speed is reduced substantially by as much as $50 \%$ [9].

\subsubsection{When we move our eyes}

Not every word is fixated during reading some words are skipped. Research suggests that skipped words are processed up to a certain extent in the parafovea. Skipped words undergo some degree of processing, even though they are not directly fixated. An experiment was conducted where the participants' eyes were tracked during reading. The words that were skipped were then deleted and another participant tried to read the passage. However, with the missing words it proved very difficult, therefore the skipped words must have been processed by the original participants, even though they were not fixated [10]. Word length is a strong predictor of whether a word will be skipped or not, with short words being skipped more often than long words $[11,12]$. Ease of processing has also been shown to influence word skipping ratios, as seen in experiments examining the effects of more high-level linguistic factors on word skipping. Highly predictable words are skipped more often than those that are not predictable [13], for example, in the sentence, "Since the wedding was today, the baker rushed the wedding cake/pies to the reception." The word cake is more predictable than the word pies and will therefore be more likely to be skipped. In addition, high frequency words are skipped more often than low frequency words [14-16]. For example, in the sentence, "Mary bought a chest/trunk despite the high price.", the high frequency word chest would be more likely to be skipped compared to the low frequency word trunk.

\subsection{Signalling}

Written text contains numerous different types of signals that the reader can make sense of and utilise to assist in their comprehension of the text, for example, including headings to break up the text content and signal the content contained within the following section or highlighting important words in the text by underlining them. Signals of this kind presumably evolved as a means for an author to guide the reader's processing of the text. The author can make the structure of the text more salient to the reader by including headings and subheadings, or make an individual word or small section more salient to the reader by underlining a word or highlighting a word by displaying it in italics or boldface.

Typological cues (such as boldface or underline) highlight the importance of a small region of text embedded within the main text and they have been shown to improve memory for the signalled content [17-20]. However, simply putting text in bold or underlining the text does not automatically mean it will be remembered. The reader has to assume what the typographical cues mean in order to utilise them. It has been found that when the reader expected "questions on specific details", underlining sections of text facilitated cued recall for those sections. However, when the reader was expected to provide an outline of text or a list of solutions to the problem discussed in the text, the readers did not experience any benefits from the signalling. The reader takes into account the purpose for why they are reading when deciding whether to utilise the signalling cues present in the text [21].

In terms of reading on the Web, hyperlinks may serve as typographical signals due to the fact that hyperlinks are a single word or short phrase that is salient from the rest of the text. Hyperlinked words could be considered as important pieces of information compared to the rest of the non-linked text and the presence of the hyperlink may add emphasis to that section of text.

\subsubsection{Saliency of Blue Hyperlinks}

One of the main differences between reading plain text and reading online is the fact that online text contains hyperlinks, which are salient in comparison to the surrounding text. Salient items are those which stand out from the rest of the items. Hyperlinks are often denoted in blue and the rest of the text is the same colour, often black. 
Nielsen [22] claimed that it is the mother of bad design conventions is the decision to make hypertext links blue' because only $2 \%$ of the cones on the retina are blue sensitive making it a poor choice in terms of usability [23]. However, Nielsen admits that the convention of the blue hyperlink should remain because users know that blue text denotes a hyperlink, making it easier for users to recognise hyperlinks more rapidly. This is supported by research on automatic attention which suggests that when a user consistently searches the same environment for the same information which is always represented in the same way the processing becomes automatic $[24,25]$. This could be true for hyperlinks because blue text in a Webpage context almost always represents a hyperlink. This effect has been observed where it was found that participants were quicker when searching for a target word in a Webpage that was blue and underlined than target words that were black and underlined, even though the black text should be easier to read in terms of usability [26].

\subsection{Skim reading}

The current literature suggests that reading on the Web is more likely to involve skim reading $[27,28]$. One of the first experiments exploring skim reading behaviour used eye movement methodology to investigate the differences in how people read when they are reading normally or reading quickly. Researchers studied three different types of reading: normal reading; skim reading; and speed reading. It was suggested that readers increase their speed by sacrificing the amount they understand from the text, thereby exhibiting a trade-off of greater speed at the cost of reduced comprehension. They found that speed readers were three times faster than normal readers and the skimmers were two and a half times faster than the normal readers in reading through the text presented to them. The eye movement analyses showed that the skimmers and speed readers fixated fewer words than the normal readers and the normal readers had longer fixations when they fixated a word [29].

Researchers have also shown a reduction in comprehension when reading rate increased. In order to explore skim reading behaviour, passages of text where displayed to participants and they were given varying amounts of time to read the passages. When testing the participants with comprehension questions, those who had the shortest time to read the text performed the worst. This suggests that by increasing reading speed, comprehension is reduced [30]. However, it is difficult to see if comprehension is reduced globally across the text. Comprehension may only be reduced on certain parts of the text and not on all parts, some parts might be considered important and the reader may focus on those parts of the text. Other researchers have tried to explore this by having independent participants rate sentences within passages of text and exploring if skim reading is used to skim over the unimportant pieces of text rather than just skim read all of the text. For example, Masson [31] manipulated the time participants had to read passages of text and tested their recognition memory for the text in the passages. The recognition rates decreased when the participants' time to read the text decreased. However, this was only true for those sentences that were rated as 'unimportant' (as judged by a different set of independent participants). In order for skimming to be an effective method of gaining information quickly, without sacrificing comprehension the reader needs to focus on the important information and not waste time on the unimportant information.

Duggan and Payne [32] conducted several experiments to test if participants focused on the more important information in the text when skim reading. They found that readers asked to skim read had better memory and comprehension performance for important details from the text, but not for the unimportant details. Skim reading is a trade-off whereby the reader is trading depth of comprehension for speed, but while trying to minimize the loss of comprehension by using an effective strategy to move through the text quickly.

\subsection{Navigation and Cognitive Load}

When reading non-Web forms of plain text, such as novels or magazine articles, the text is written in such a way that it is best comprehended when read from the beginning to end in a linear fashion. Webpages, on the other hand, are an interconnected network of text passages that do not necessarily need to be read in a specific order, nor do they tend to have a specified beginning and end point [33].

There is an on-going debate about whether in-text hyperlinks hinder reading. Carr [34] suggested that hyperlinks within the text are a distraction and therefore hinder comprehension of the text. He argued that having to evaluate hyperlinks and navigating a path through them is demanding. Carr goes on to suggest that deciphering hypertext substantially increases readers' cognitive load and weakens their ability to comprehend and retain what they are reading.

There is also research that explores working memory and the concept of cognitive load and its impact on reading hypertext. DeStefano and LeFevre [35] conducted a review of cognitive load in relation to reading hypertext. They argued that the extra task demands of reading hypertext causes an increased cognitive load to the readers in comparison to linear text. Because the readers 
have to make decisions about which hyperlinks to follow as an additional task to reading.

Recently, Scharinger, Kammerer and Gerjets [36] measured both the EEG and pupil size of readers engaging in a task that closely simulated hypertext reading and link selection. They found evidence of increased load on executive functions when the reader had to perform hyperlink-like selection. This suggests that there is additional cognitive load when having to make hyperlink-like selections which might hamper reading and comprehension.

It is not just the decision of whether or not to click a hyperlink that could increase cognitive load. Whenever a reader chooses to follow a hyperlink and explore different content, this could interrupt the on-going comprehension processes. Comprehension involves the creation and development of situation models, which are complex mental representations that the reader forms to integrate statements in the text they are reading into their knowledge [37]. This is also observed by Dee-Lucas and Larkin [38] who found that hyperlinks in text distract users by interrupting information processing. While reading, users may stop to click on hyperlinks in the middle of text content, thus interrupting their cognitive processing and leaving the reader with a fragmented representation of the text content.

\section{INITIAL RESULTS}

\subsection{Saliency of Hyperlinks}

The present experiments focus on how we read hyperlinked text and whether hyperlinks influence reading behaviour. In order to examine how hyperlinks affect reading behaviour, we first need to find out if any disruption of reading occurs and decide whether it is due to the hyperlink being a salient colour, or because the blue text of the hyperlink is perceived as being important due to the additional information that it can link to.

Three experiments were conducted to explore this issue. The first experiment, Experiment $1 \mathrm{~A}$, explored whether salient, coloured words negatively impact reading behaviour. Before examining what the impact is of hyperlinks coloured in blue in a hypertext environment, it is important to examine the impact of reading a word in blue in a sentence outside of a hypertext context. Experiment $1 \mathrm{~A}$ only used a single coloured word in a single-line sentence to explore the impact of saliency. Therefore, a follow-on experiment was conducted exploring whether multiple coloured words had an impact on reading, this was Experiment 1B.

The third experiment, Experiment 1C, explored whether perceiving the words as hyperlinks impairs reading behaviour. We also implemented a word frequency manipulation in order to explore whether common lexical effects are present in hyperlinked text and to investigate if they are modulated by the word being hyperlinked. Together, the experiments assessed whether there is a difference between reading coloured words and reading hyperlinks and how this affects reading behaviour. Experiment $1 \mathrm{~A}$ and 1B will help us to separate whether any observed effects seen in Experiment $1 \mathrm{C}$ are due to the saliency of a blue word or due to the fact that the blue words are in a hypertext environment.

Experiment $1 \mathrm{~A}$ demonstrated that a coloured word is less likely to be skipped, but that making a word coloured does not negatively impact reading behaviour unless the colour has reduced contrast (e.g. grey text) making it difficult to read.

Experiment 1B demonstrated that when a coloured word or words are present in the text they have no negative impact on reading behaviour. We observed in Experiment $1 \mathrm{~A}$ a reduction in skipping from the single coloured target word. This was presumably because a single coloured word in a sentence works as a signal of importance to that particular word. When there are multiple words being highlighted in the sentence this reduces the signal and therefore we did not observe the same reduction in skipping.

Experiment $1 \mathrm{C}$ demonstrated that hyperlinked words are processed differently to coloured words. There was a main effect of word frequency where low frequent words had longer fixation times than high frequent words. This frequency effect was also qualified by an interaction with whether the target word was hyperlinked or unlinked. There were significantly longer reading times and more rereading when the target word was hyperlinked and low frequent. In Experiment $1 \mathrm{C}$ there was no difference in skipping rates when the word was hyperlinked vs when it was unlinked. This suggests that there is a difference between the visual appearance of a coloured word and when it is perceived as a hyperlink. We expect this is because in a Web environment the reader knows that a blue word indicates a hyperlink and they are quite common in the text. Whereas in a single line sentence the stand alone coloured word may seem more important a signal when it is the only word coloured for no special reason.

\subsection{Importance of Hyperlinks}

Experiment 2 involved participants reading edited Wikipedia articles on a computer screen and then judging each sentence based on how important it was for the general meaning of the content on that particular page. Each sentence was given an importance rating between 1 (Not very important) 5 (Very important). The results from these importance ratings were used to understand what 
the reader judges as "important" and explore whether the presence of hyperlinks affects the importance ratings of the sentences. The results from Experiment 2 fed into Experiment 3 . The results suggest that there are a number of factors that influence how important a sentence is judged: sentence length (longer rated as more important), position on page (nearer the top rated as more important) and number of hyperlinks (more hyperlinks rated as more important) all contributed to the rated importance of a sentence.

\subsection{Skim reading on the Web}

Experiment 3 focuses on how hyperlinks impact on skim reading behaviour and how individuals sample the text and extract information from it. With the large amount of information online some have argued that skim reading is a common behaviour when reading on the Web $[27,28]$. It is an efficient way of gaining as much information as possible in the shortest amount of time, while trying not to sacrifice comprehension. Hyperlinks may be used to assist in the strategy of determining what parts of the text contain important information and what should be read to gain comprehension relevant to the reader's goal.

Experiment 3 explored the differences between reading for comprehension and skim reading on reading behaviour by recording the participants' eye movements. The ratings from Experiment 2 were also used to examine the impact of the importance that was given to each sentence. The two most important and two least important sentences from each edited Wikipedia article were selected and comprehension questions were created based on these sentences. From the comprehension accuracy variable we can then separate the comprehension of the important and unimportant sentences.

Experiment 3 demonstrated that skim reading does have an influence on reading behaviour. As observed in the eye movement measures, fixations were shorter on average for the skim reading condition, replicating the findings of Just and Carpenter [29]. However, the most interesting finding here is in relation to the impact that hyperlinks have on reading behaviour. In Experiment $1 \mathrm{C}$ we found that hyperlinks had a limited impact on reading behaviour, apart from increased re-reading of the low frequency, hyperlinked words. We did replicate the finding that when reading normally, hyperlinks are not a hindrance to reading.

During skim reading, hyperlinked words were less likely to be skipped and they were more likely to be the words that were fully processed. This is shown by the presence of a frequency effect being observed in the hyperlinked words for skim reading, but not for the unlinked words. We suggest that the reason for the lack of a frequency effect in the unlinked words is because they were not being fully processed.

In terms of comprehension, accuracy for the comprehension questions was reduced when the participants were skim reading. However, the participants showed marginally better accuracy for the comprehension questions about the important questions compared to the unimportant questions. This suggests that the readers were engaging in an adaptive strategy to try and focus on the important information in the text.

\subsection{Navigation and Reading on the Web}

In Experiment 4 the readers were allowed to read and navigate the Web environment. It has been suggested that the additional task of decision making could negatively impact reading behaviour. Therefore, the analysis in this section focuses on how the decision to click impacts on reading.

Preliminarily findings suggest that the reader engages in 3 phases of behaviour when reading a Webpage. A first phase where they scan the content very quickly and look to the bottom of the page and back to the top, almost as if they are checking the content and if it has hyperlinks present before properly engaging to read the text. Next the reader reads the text in a linear fashion top to bottom. The last phase involves a scan of the page again and making a decision about what hyperlink to click to navigate to a new Webpage.

Further analyses will explore how being able to click the hyperlinks impacts on reading behaviour and we will explore the cognition that takes place during the three phases we have identified.

\section{MAIN CONTRIBTIONS}

The goal of examining how we read, interact with and process hypertext here is motivated by both practical and theoretical interests. Looking at real world tasks can inspire theoretical questions that serve as a motivation for novel empirical questions. To date, previous research that has examined reading behaviour has focused mainly on reading for comprehension, and the theoretical and computational models of reading typically model the reading of a single line of text rather than passages of text. The majority of this work also tends to focus on lexical processing rather than higher level issues such as discourse processing or task effects (how different tasks influence how people perform a task). Exploring how people read hypertext would explore these higher level issues such as task effects (by comparing it with different tasks) and how the high level information of knowing that a hyperlink links a word or phrase to another piece of content affects the reader. 
Hypertext and the Web therefore offer an ideal task/stimulus space for extending existing models and theories by allowing for an examination of those models and theories under new, but related conditions. Furthermore, by studying how people read hypertext on the Web, it will then be possible to generate suggestions for how to design and setup Web pages in a way that most suits the demands and limitations of the human information processing system.

\section{REFERENCES}

[1]W. H. Dutton and G. Blank, "Next generation users: The Internet in Britain. Oxford Internet Survey 2011.," Oxford Internet Institute, University of Oxford, 2011.

[2]K. Rayner, "Eye movements in reading and information processing: 20 years of research.," Psychol. Bull., vol. 124, no. 3, pp. 372-422, Nov. 1998.

[3]K. Rayner, "Eye movements and attention in reading, scene perception, and visual search," Q. J. Exp. Psychol., vol. 62, no. 8, pp. 14571506, Aug. 2009.

[4]T. H. Nelson, "A file structure for the complex, the changing, and the indeterminate," in ACM 20th National Conference, 1965, pp. 84-100.

[5]K. Rayner, "Eye movements in reading and information processing.," Psychol. Bull., vol. 85, no. 3, pp. 618-60, May 1978.

[6]S. P. Liversedge and J. M. Findlay, "Saccadic eye movements and cognition," Trends Cogn. Sci., vol. 4, no. 1, pp. 6-14, Jan. 2000.

[7]I. D. Gilchrist, V. Brown, and J. M. Findlay, "Saccades without eye movements," Nature, vol. 390, no. 6656, pp. 130-131, 1997.

[8]A. L. Yarbus, Eye movements and vision. New York: Plenum Press, 1967.

[9]R. K. Morris, K. Rayner, and A. Pollatsek, "Eye movement guidance in reading: The role of parafoveal letter and space information," J. Exp. Psychol. Hum. Percept. Perform., vol. 16, no. 2, pp. 268-281, 1990.

[10] D. F. Fisher and W. L. Shebilske, "There is more that meets the eye than the eyemind assumption," in Eye movements and human information processing, R. Groner, G. McConkie, and C. Menz, Eds. Amsterdam: North-Holland, 1985, pp. 149-158.
[11] M. Brysbaert, D. Drieghe, and F. Vitu, "Word skipping: Implications for theories of eye movement control in reading," in Cognitive processes in eye ..., G. Underwood, Ed. Oxford, UK: Oxford University Press, 2005, pp. 53-78.

[12] K. Rayner and G. McConkie, "What guides a reader's eye movements?," Vision Res., vol. 16, no. 8, pp. 829-837, 1976.

[13] D. Balota, A. Pollatsek, and K. Rayner, "The interaction of contextual constraints and parafoveal visual information in reading.," Cogn. Psychol., vol. 17, no. 3, pp. 364-90, Jul. 1985.

[14] J. Henderson and F. Ferreira, "Effects of foveal processing difficulty on the perceptual span in reading: implications for attention and eye movement control.," J. Exp. Psychol. Learn. Mem. Cogn., vol. 16, no. 3, pp. 417-29, May 1990.

[15] A. W. Inhoff and K. Rayner, "Parafoveal word processing during eye fixations in reading: Effects of word frequency," Percept. Psychophys., vol. 40, no. 6, pp. 431-439, 1986.

[16] K. Rayner and M. H. Fischer, "Mindless reading revisited: Eye movements during reading and scanning are different," Percept. Psychophys., vol. 58, no. 5, pp. 734-747, 1996.

[17] V. Cashen and K. Leicht, "Role of the isolation effect in a formal educational setting.," J. Educ. Psychol., vol. 61, no. 6, pp. 484-486, 1970.

[18] J. Crouse and P. Idstein, "Effects of encoding cues on prose learning.," J. Educ. Psychol., vol. 83, no. 4, pp. 309-313, 1972.

[19] R. L. Fowler and A. S. Barker, "Effectiveness of highlighting for retention of text material," J. Appl. Psychol., vol. 59, no. 3, pp. 358-364, 1974.

[20] R. F. Lorch, E. P. Lorch, and M. Klusewitz, "Effects of typographical cues on reading and recall of text," Contemp. Educ. Psychol., vol. 20, pp. 51-64, 1995.

[21] J. Golding and S. Fowler, "The limited facilitative effect of typographical signals," Contemp. Educ. Psychol., vol. 13, pp. 99-113, 1992.

[22] J. Nielsen, "When bad design elements become the standard," 1999.

[23] W. O. Galitz, The essential guide to user interface design: An introduction to GUI design principles and techniques. New York, NY: John Wiley \& Sons, 1997.

[24] W. Schneider and R. M. Shiffrin, "Controlled and automatic human information 
processing: I. Detection, search, and attention," Psychol. Rev., vol. 84, no. 1, pp. 1-66, 1977.

[25] R. M. Shiffrin and W. Schneider, "Controlled and automatic human information processing: II. Perceptual learning, automatic attending and a general theory," Psychol. Rev., vol. 84, no. 2, pp. 127-190, 1977.

[26] C. S. Campbell and P. P. Maglio, "Facilitating navigation in information spaces: Road-signs on the World Wide Web," Int. J. Hum. Comput. Stud., vol. 50, no. 4, pp. 309327, 1999.

[27] Z. Liu, "Reading behavior in the digital environment: Changes in reading behavior over the past ten years," J. Doc., vol. 61, no. 6, pp. 700-712, 2005.

[28] J. Morkes and J. Nielsen, "Concise, SCANNABLE, and Objective: How to write for the Web," 1997.

[29] M. A. Just and P. A. Carpenter, The Psychology of Reading and Language Comprehension. Newton, MA: Allyn and Bacon, 1987.

[30] R. P. Carver, "Rauding theory predictions of amount comprehended under different purposes and speed reading conditions," Read. Res. Q., vol. 19, no. 2, pp. 205-218, 1984.

[31] M. E. J. Masson, "Cognitive processes in skimming stories.," J. Exp. Psychol. Learn. Mem. Cogn., vol. 8, no. 5, pp. 400-417, 1982.

[32] G. B. Duggan and S. J. Payne, "Text skimming: The process and effectiveness of foraging through text under time pressure.," J. Exp. Psychol. Appl., vol. 15, no. 3, pp. 228-242, 2009.

[33] J. M. Slatin, "Reading hypertext: Order and coherence in a new medium," Coll. English, vol. 52, no. 8, pp. 870-883, 1990.

[34] N. G. Carr, The Shallows. New York: W. W. Norton, 2010.

[35] D. DeStefano and J. LeFevre, "Cognitive load in hypertext reading: A review," Comput. Human Behav., vol. 23, pp. 1616-1641, May 2007.

[36] C. Scharinger, Y. Kammerer, and P. Gerjets, "Pupil dilation and EEG alpha frequency band power reveal load on executive functions for link-selection processes during text reading," PLoS One, vol. 10, no. 6, pp. 1-24, 2015.

[37] W. Kintsch, "The role of knowledge in discourse comprehension: A constructionintegration model," Psychol. Rev., vol. 95, no. 2, pp. 163-182, Apr. 1988.
[38] D. Dee-Lucas and J. H. Larkin, "Learning from electronic texts: effects of interactive overviews for information access," Cogn. Instr., vol. 13, no. 3, pp. 431-468, 1995. 